\title{
Reliability of near-infrared spectroscopy measures of cerebral oxygenation and blood volume during handgrip exercise in nondisabled and traumatic brain-injured subjects
}

\author{
Yagesh Bhambhani, PhD; ${ }^{1 *}$ Ram Maikala, PhD; ${ }^{1}$ Mamdouh Farag, BSc; ${ }^{1}$ Gary Rowland, MSc ${ }^{1-2}$ \\ ${ }^{1}$ Faculty of Rehabilitation Medicine, University of Alberta, Edmonton, Alberta, Canada; ${ }^{2}$ Brain Injury Rehabilitation \\ Program, Alberta Hospital Ponoka, Ponoka, Alberta, Canada
}

\begin{abstract}
We compared the test-retest reliability of nearinfrared spectroscopy (NIRS) measures of cerebral oxygenation and blood volume during a rhythmic handgrip exercise in 13 nondisabled subjects and 25 subjects with moderate to severe traumatic brain injury (TBI). Subjects with TBI (average Glasgow Coma Scale score $=4.2$, average time since injury $=21 \mathrm{mo}$ ) had completed an acute brain injury rehabilitation program. After 2 min of rest, each subject performed $60 \mathrm{~s}$ of maximal rhythmic handgrip contractions with the right hand in two trials 24 to $48 \mathrm{~h}$ apart. We used NIRS to measure cerebral oxygenation and blood volume responses from the left prefrontal lobe. Both groups' cerebral oxygenation and blood volume increased during handgrip contractions. The change in cerebral oxygenation was significantly lower in subjects with TBI compared with nondisabled subjects. Intraclass correlations between the two trials for cerebral oxygenation and blood volume were 0.83 and 0.80 , respectively, in nondisabled subjects and 0.70 and 0.64 , respectively, in subjects with TBI. The findings indicate that NIRS is a reliable noninvasive technique for evaluating cerebral oxygenation and blood volume changes during motor function. NIRS can be useful in monitoring recovery of cerebral oxygenation during rehabilitation of patients with TBI.
\end{abstract}

Key words: Bland-Altman analysis, blood volume, cerebral oxygenation, handgrip contractions, intraclass correlations, motor function, near infrared spectroscopy, rehabilitation, testretest reliability, traumatic brain injury.

\section{INTRODUCTION}

Near-infrared (NIR) spectroscopy (NIRS) is a valid noninvasive optical method of measuring relative changes in cerebral oxygenation and blood volume in humans [1-2]. In cerebral tissue, the two main chromophores (light-absorbing compounds) are hemoglobin $(\mathrm{Hb})$ and cytochrome oxidase. NIRS is based on the differential absorption properties of these chromophores in the NIR range, i.e., between 700 and $1,000 \mathrm{~nm}$. At $760 \mathrm{~nm}, \mathrm{Hb}$ occurs primarily in the deoxygenated state (deoxyHb), whereas at $850 \mathrm{~nm}$, it occurs in the oxygenated state $(\mathrm{oxyHb})$. Hence, by monitoring the difference in absorbency between these two wavelengths, one can evaluate the degree of tissue deoxygenation. This change reflects the balance between the oxygen supply at the

Abbreviations: BOLD = blood oxygen level-dependent, deoxyHb = deoxygenated hemoglobin, fMRI = functional magnetic resonance imaging, $\mathrm{Hb}=$ hemoglobin, ICC $=$ intraclass correlation coefficient, NIR $=$ near infrared, NIRS $=$ near-infrared spectroscopy, $\mathrm{OD}=$ optical density, oxyHb $=$ oxygenated hemoglobin, $\mathrm{SD}=$ standard deviation, $\mathrm{TBI}=$ traumatic brain injury .

*Address all correspondence to Dr. Yagesh Bhambhani, Faculty of Rehabilitation Medicine, Corbett Hall, Room 373, University of Alberta, Edmonton, Canada T6G 2G4; 780-492-7248; fax: 780-492-1626.

Email: yagesh.bhambhani@ualberta.ca

DOI: 10.1682/JRRD.2005.09.0151 
level of the small blood vessels (i.e., the arterioles, capillaries, and venules) and the amount of oxygen extracted by the tissue. The change in the sum of the absorbency signals (deoxyHb + oxyHb) at the 760 and $850 \mathrm{~nm}$ wavelengths is an index of the change in localized blood volume. One limitation of continuous wave NIRS is that absolute oxygen uptake and blood volume changes of the tissue cannot be quantified because the path length of the signal cannot be determined [1].

The validity of NIRS in evaluating changes in jugular bulb venous oxygen saturation, which is considered an accurate index of mixed cerebral oxygenation, has been established at varying levels of hypoxia in anesthetized humans [3]. As well, evidence indicates that the alteration in cerebral oxygenation and blood volume during hand function corresponds closely with the blood oxygen level-dependent (BOLD) changes evident during functional magnetic resonance imaging (fMRI) [4-5]. The test-retest reliability of cerebral NIRS measurements has been established in nondisabled males and females of different ages under various experimental conditions, including postural changes [6-8], cognitive stimulation and hyperventilation [9], and carbon dioxide breathing [10]. As well, the reproducibility of these responses during symptom-limited incremental exercise has been demonstrated in patients with heart failure [11].

During the last decade, researchers have used NIRS extensively to evaluate cerebral oxygenation and blood volume during various tasks involving cognitive, visual, auditory, and motor stimulation [12-16]. The primary advantage of NIRS over other techniques such as fMRI in evaluating cerebral function is that it provides information on oxyHb, deoxyHb, and total $\mathrm{Hb}$ with a high temporal resolution at the microvascular level. This information is biochemically specific and provides a more comprehensive evaluation of the cerebral hemodynamic response during functional tasks [7,9]. As well, because of the cost effectiveness, portability, and ease of use of NIRS, it is an attractive noninvasive technique for evaluating cerebral function in a variety of experimental conditions and settings. Several investigators have used NIRS to evaluate cerebral oxygenation and blood volume changes in patients with neurological disorders, including traumatic brain injury (TBI) [17-20], stroke [21], brain tumors [22-23], and cerebral ischemia [23]. The clinical application of NIRS in patients with severe acute TBI has indicated that the technique must be used cautiously because of its limited sensitivity [18] and oscillations that are observed in the oxyHb signal [19]. Buchner et al. reported that NIRS measurements were possible in only 80 percent of 31 patients with acute severe TBI due to a subarachnoid hemorrhage or a severe head injury, whereas good quality data were obtained from these same patients by other invasive techniques [18]. However, other evidence has suggested that NIRS is useful for evaluating changes in cerebral hemodynamics in children and adults with chronic severe TBI and could provide additional insight into the pathophysiology of the injury $[17,20]$. Saitou et al. documented acute changes in the cerebral oxygenation and blood volume responses in stroke patients during 13 different cognitive, visual, and motor tasks and suggested that NIRS could be used to monitor the progress of stroke patients' rehabilitation [21]. Sakatani et al. reported that the evoked changes in cerebral blood oxygenation as measured by NIRS during handgrip tasks significantly differed between nondisabled subjects and patients with cerebral ischemia and brain tumors [22]. Fujiwara et al. reported that the increases in frontal lobe cerebral oxygenation and blood volume in patients with brain tumors performing handgrip tasks were significantly lower on the lesion compared with the nonlesion side [23].

Many patients with neurological disorders experience cognitive and neuropsychological deficits that could affect their ability to perform daily functional tasks. Therefore, clinicians responsible for the rehabilitation of such patients must be able to evaluate alterations in cerebral hemodynamics during these functional tasks to monitor the patients' progress [21]. To our knowledge, cerebral hemodynamic alterations as a result of rehabilitation have not been demonstrated with this use of NIRS in patients with neurological disorders. However, before these alterations can be examined confidently, the reliability of NIRS in the TBI patient population must be demonstrated. Therefore, the purposes of this study were to (1) examine the test-retest reliability of NIRS in measuring cerebral oxygenation and blood volume changes during maximal rhythmic handgrip contractions in nondisabled subjects and subjects with moderate to severe chronic TBI and (2) compare the responses between the two subject groups. We hypothesized that (1) cerebral oxygenation and blood volume changes would be reproducible in both subject groups and (2) the magnitude of these changes would be significantly lower in the subjects with TBI compared with their nondisabled counterparts. 


\section{METHODS}

\section{Subjects}

Written informed consent was obtained from 13 nondisabled subjects (8 males and 5 females) and 25 subjects with moderate to severe TBI (21 males and 4 females). The nondisabled subjects were undergraduate and graduate students at the University of Alberta (Edmonton, Alberta, Canada). They were all recreationally active and had no medical disorders. The subjects with TBI were inpatients at the Brain Injury Rehabilitation Program at Alberta Hospital Ponoka (Ponoka, Alberta, Canada). Details of this long-term rehabilitation program for slowto-recover patients with moderate to severe TBI have been previously reported [24-25]. The inclusion criteria for the subjects with TBI were (1) a medically stable condition, (2) completion of acute brain injury rehabilitation, (3) sufficient functional capacity in the right hand to grip and squeeze a dynamometer, and (4) approval from the attending physician for participation in the study. After obtaining the subjects' informed consent, we obtained the Glasgow Coma Scale scores [26] at the time of injury from the medical records of the subjects with TBI. Both subject groups then completed the testing protocols that were approved by the Regional Health Research Ethics Board and are described subsequently.

\section{Experimental Design and Testing Protocol}

Both subject groups were initially familiarized with the equipment and testing procedures before undertaking any measurements. Thereafter, each subject reported to the testing laboratory on two separate occasions that were 24 to 48 hours apart. Testing was conducted in a quiet room at approximately the same time of the day, usually between 9 a.m. and 1 p.m. and at least 1 hour postmeals. At the start of the testing session, the maximal grip strength of the right hand was recorded in duplicate with a hand-grip dynamometer (Model 78010, Lafayette Instruments, Lafayette, Indiana) that weighed $1 \mathrm{~kg}$. In the nondisabled subjects, we recorded the grip strength in the sagittal plane while the subject was sitting down with the right elbow fully extended and the arm abducted $90^{\circ}$. The subjects with TBI adopted a similar posture whenever possible. However, several subjects were unable to maintain this posture and therefore the measurement was taken in a posture that was most comfortable to them, typically with the arm abducted between $45^{\circ}$ and $90^{\circ}$. After we recorded grip strength, we prepared the subject for the NIRS measurements, in which cerebral oxygenation and blood volume measurements were recorded continuously during the following test protocol: 2 minutes of rest (baseline), 1 minute of intermittent maximal handgrip contractions with the right arm, and then 2 minutes of recovery. These intermittent handgrip contractions were performed in the posture just described. The subjects squeezed the dynamometer maximally at a frequency of 10 contractions per minute. Each 3-second contraction was interspersed with 3 seconds of relaxation. We used this procedure to elicit maximal cerebral stimulation during the exercise phase. During the resting and recovery phases, the subjects were asked to mentally relax and avoid any unnecessary movement.

\section{Near-Infrared Spectroscopic Measurements}

The cerebral oxygenation and blood volume responses were continuously monitored by a dual-wavelength NIRS (Model MRM91, MicroRunman, NIM Inc, Philadelphia, Pennsylvania). This instrument consisted of three units: (1) a light source with a tungsten light-emitting lamp placed approximately $4 \mathrm{~cm}$ from two silicone diodes that absorb light at 760 and $850 \mathrm{~nm}$, (2) a recording device that interfaced with a computer, and (3) NIR communication software (NIM Inc) that calculated the cerebral oxygenation and blood volume values using standardized algorithms (described subsequently). The light source penetration depth was approximately $2.5 \mathrm{~cm}$, which was sufficient to reflect from cerebral gray matter. The probe was placed on the left prefrontal lobe approximately $3 \mathrm{~cm}$ from the midline of the forehead, just above the supraorbital ridge [13]. It was secured on the forehead with a dark tensor bandage so that background light would not affect the signal. Pilot testing with this light source in different positions indicated that this placement yielded a strong NIRS signal and was sensitive to changes during static exercise.

The NIRS instrument was calibrated individually for each subject using the NIR communication software. For the calibration procedure, a deep penetration setting with the light intensity ranging from 100 to $110 \mathrm{mV}$ was used for most subjects. Similar probe positions and calibration settings were used for both test trials. Although the instrument calibrated properly in all cases, in some instances obtaining the cerebral oxygenation and blood volume measurements was difficult once the test was initiated. In such cases, we moved the position of the light source by 0.5 to $1.0 \mathrm{~cm}$ as previously described [13] and 
recalibrated the NIRS to obtain these measurements. The NIRS data were collected at a $1 \mathrm{~Hz}$ frequency and stored online for subsequent analysis. From the raw NIRS data, we calculated the optical density (OD) at 760 and $850 \mathrm{~nm}$ by applying the modified Beer-Lambert law as follows [1]: OD $=a c d B+G$, where $a=$ absorption coefficient of the tissue, $c=$ concentration of the tissue, $d=$ distance between optodes on the measuring probe, $B=$ differential path length factor of the tissue, and $G=$ geometry of the tissue. The difference in OD between the two wavelengths indicated the relative change in cerebral oxygenation, while the sum of the OD at the two wavelengths indicated the relative change in blood volume. The real time data were averaged over 5-second intervals during each phase of the trial. Previous fMRI research has demonstrated that anticipation of a task can increase the cerebral NIRS responses [27]. Therefore, for each subject and each trial, we calculated the delta values of the cerebral oxygenation and blood volume responses as the difference between the peak 5-second value observed during the 60 -second handgrip contraction and the last 5-second resting value recorded prior to the initiation of muscle contractions.

\section{Statistical Analysis}

The mean values of the subject characteristics were compared using independent $t$-tests. Normality of the cerebral oxygenation and blood volume responses in the two subject groups was examined with the KolmogorovSmirnov test. We used a two-way analysis of variance (group $\times$ trial) to examine the differences between the two repeated trials in the nondisabled subjects and subjects with TBI [28]. We used the intraclass correlation coefficient (ICC) to calculate the test-retest reliability of the grip strength, cerebral oxygenation, and blood volume responses of the two subject groups. We verified the reliability of the NIRS responses in the two groups using the Bland-Altman analysis [29]. This analysis was done by plotting the differences between the two trials of each subject against the mean value of the two trials. Data points outside the 95 percent confidence interval $( \pm 1.96$ standard deviation [SD] of the mean difference) were considered outliers. We used Pearson correlation to examine the common variance and derive the regression equations between the two trials. Values were considered significant at 0.05 . All statistical analyses were performed using Statistical Package for the Social Sciences software, Version 12 (SPSS, Inc, Chicago, Illinois).

\section{RESULTS}

\section{Subject Characteristics}

Some pertinent physical characteristics of the two subject groups are given in Table 1. The mean \pm SD Glasgow Coma Scale score of the subjects with TBI was $4.2 \pm$ 1.5 (range $=3$ to 7 ), indicating that they had incurred moderate to severe injuries. At the time of testing, the subjects' mean \pm SD time since injury was $21 \pm 8$ months (range $=3$ to 84 months). The nondisabled subjects and subjects with TBI did not significantly differ in age, height, body mass, or body mass index. However, grip strength was significantly lower in the subjects with TBI compared with the nondisabled subjects.

\section{Cerebral Oxygenation and Blood Volume Trends During Handgrip Contractions in Nondisabled and TBI Subjects}

The cerebral NIRS trends during the three phases of the test protocol for a representative nondisabled subject and a subject with TBI are illustrated in Figure 1(a) and (b), respectively. During the baseline phase, cerebral oxygenation and blood volume remained fairly stable or increased slightly in both subject groups. Upon initiation of the handgrip contractions, cerebral oxygenation and blood volume increased systematically in most subjects. However, some of the subjects with TBI showed a small decline initially, but as the contractions proceeded, had a significant increase above the resting baseline value in all cases until the contractions were terminated. Approximately 20 percent $(n=7)$ of the subjects in the two groups demonstrated a small decline in these variables before the end of the 1-minute contraction period. In both subjects groups, the cerebral oxygenation and blood

\section{Table 1.}

Physical characteristics of nondisabled subjects ( $n=13 ; 8$ males, 5 females) and subjects with traumatic brain injury (TBI) $(n=25$; 21 males, 4 females). Data presented as mean \pm standard deviation.

\begin{tabular}{lll}
\hline \multicolumn{1}{c}{ Variable } & Nondisabled & \multicolumn{1}{c}{ TBI } \\
\hline Age $(\mathrm{yr})$ & $31.5 \pm 4.5$ & $31.6 \pm 9.8$ \\
Height $(\mathrm{m})$ & $1.77 \pm 0.09$ & $1.73 \pm 0.07$ \\
Body Mass $(\mathrm{kg})$ & $71.5 \pm 6.7$ & $74.1 \pm 18.9$ \\
Body Mass Index & $22.8 \pm 3.6$ & $24.5 \pm 5.5$ \\
Grip Strength (kg) & $39.6 \pm 6.6^{*}$ & $24.4 \pm 11.6$ \\
Injury (mo) & - & $21 \pm 8$ \\
Glasgow Coma Scale Score & - & $4.2 \pm 1.5$ \\
\hline${ }^{*} p<0.05$ between groups. & & \\
\hline \hline
\end{tabular}


(a)
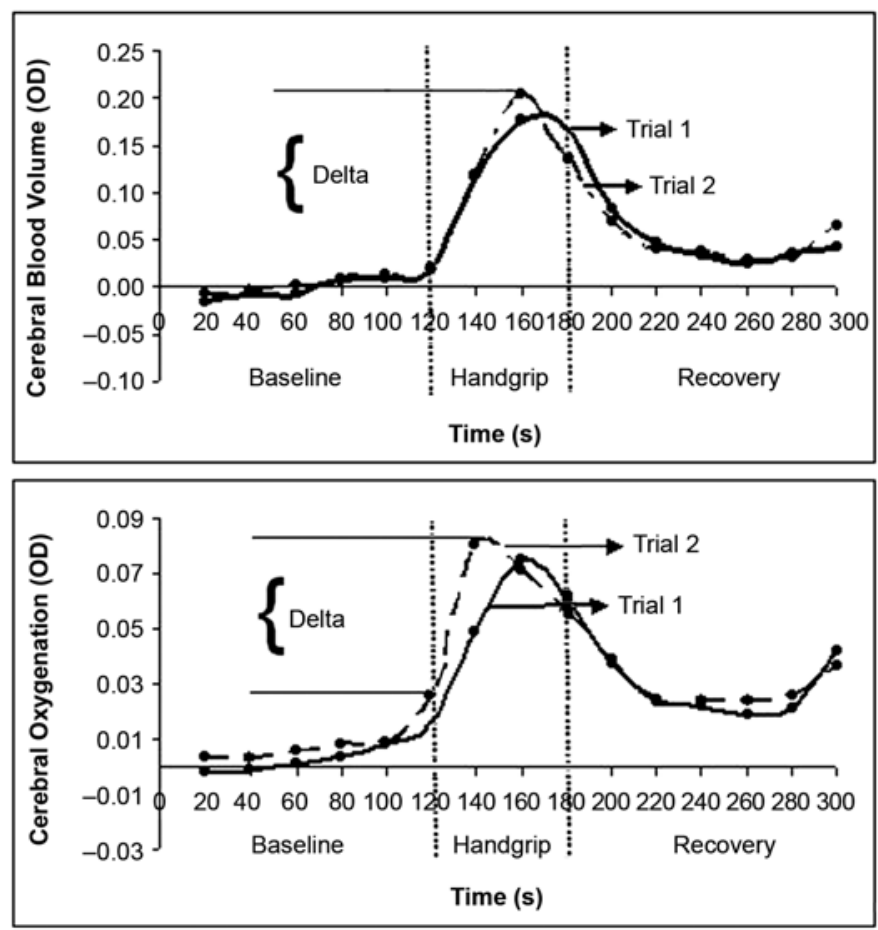

(b)
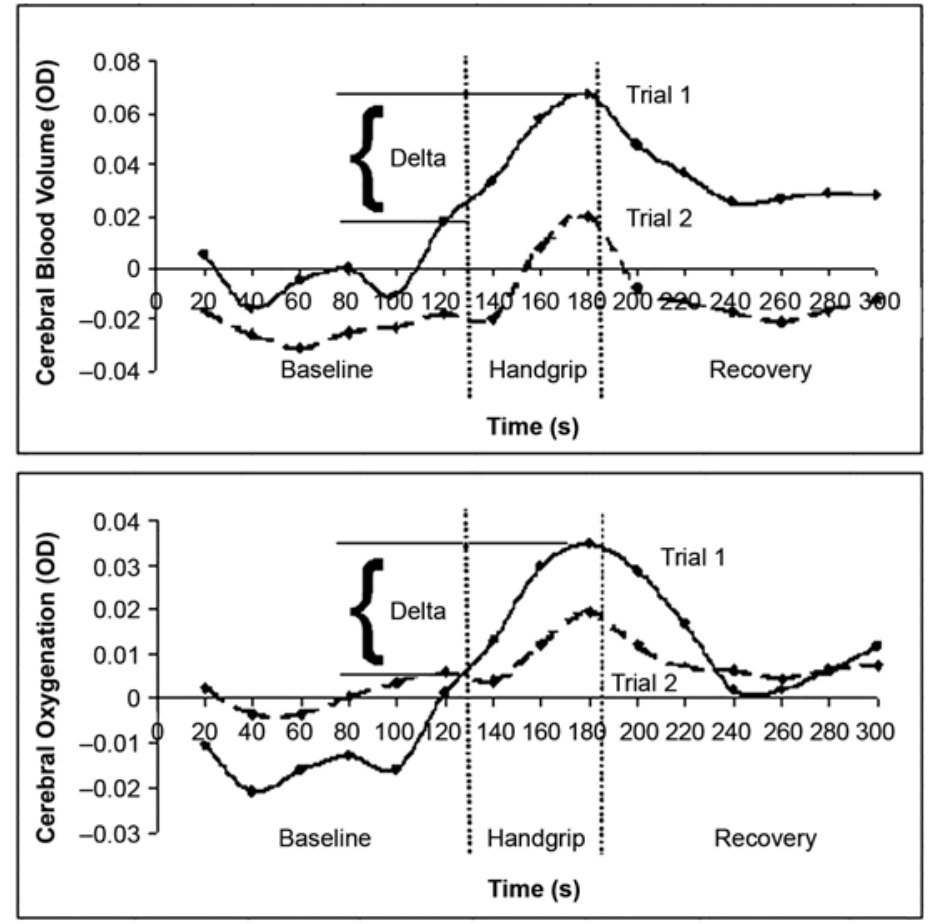

Figure 1.

Cerebral blood volume and cerebral oxygenation trends during two maximal intermittent handgrip contraction trials in representative (a) nondisabled subject and (b) subject with traumatic brain injury (TBI). Note greater difference between the two trials in subject with TBI compared with nondisabled subject. OD = optical density.

volume trends paralleled each other. However, the magnitude of change in cerebral blood volume was greater than that observed for cerebral oxygenation. During recovery, two distinct NIRS response patterns were evident. In 85 percent $(n=32)$ of the subjects, cerebral oxygenation and blood volume returned quite rapidly to the resting baseline values recorded prior to the contraction phase and stabilized during the final 30 seconds of recovery. In some of these subjects, an undershoot was evident, i.e., the values decreased to below the baseline levels. In the remaining 15 percent $(n=6)$, cerebral oxygenation and blood volume increased upon cessation of the handgrip contractions for 20 to 30 seconds and then returned to the resting baseline values. None of these subjects demonstrated an undershoot in these responses.

\section{Reliability of Cerebral Oxygenation and Blood Volume Changes in Nondisabled and TBI Subjects}

The ICCs for the cerebral oxygenation and blood volume responses in the 13 nondisabled subjects were 0.83 and 0.80 , respectively ( $p=0.002$ for each variable). Corresponding ICC values for the 25 subjects with TBI were slightly lower: 0.70 and 0.64 , respectively ( $p<0.001$ for each variable), while those for the pooled values of the nondisabled subjects and subjects with TBI $(n=38)$ were 0.76 and 0.78 , respectively ( $p<0.001$ for each variable). The Bland-Altman plots for the cerebral oxygenation and blood volume responses in the nondisabled subjects and subjects with TBI are illustrated in Figure 2(a) and (b), respectively. For the nondisabled subjects, all the data points were at or within the 95 percent confidence intervals for both variables. However, for the subjects with TBI, we noted two outliers for the change in cerebral oxygenation and one outlier for the change in cerebral blood volume during the handgrip contractions. When these outliers were removed, the ICCs were 0.73 and 0.66 , respectively ( $p<0.001$ for each variable).

The scatterplots, common variance, and regression equations for the cerebral oxygenation and blood volume responses during the two trials for the two subject groups are presented in Figure 3. For the nondisabled subjects, the common variance between the two trials for the cerebral oxygenation and blood volume were 76 and 56 percent, respectively. The corresponding values in the subjects with 
(a)
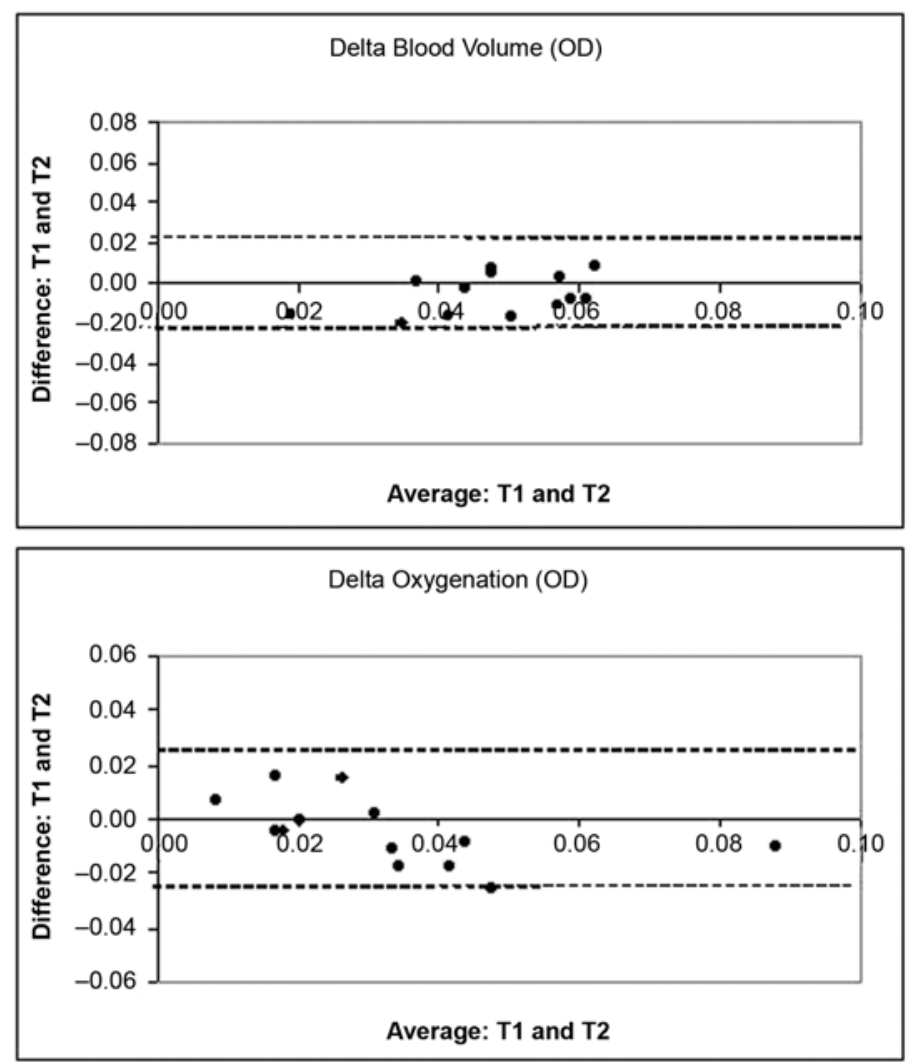

(b)
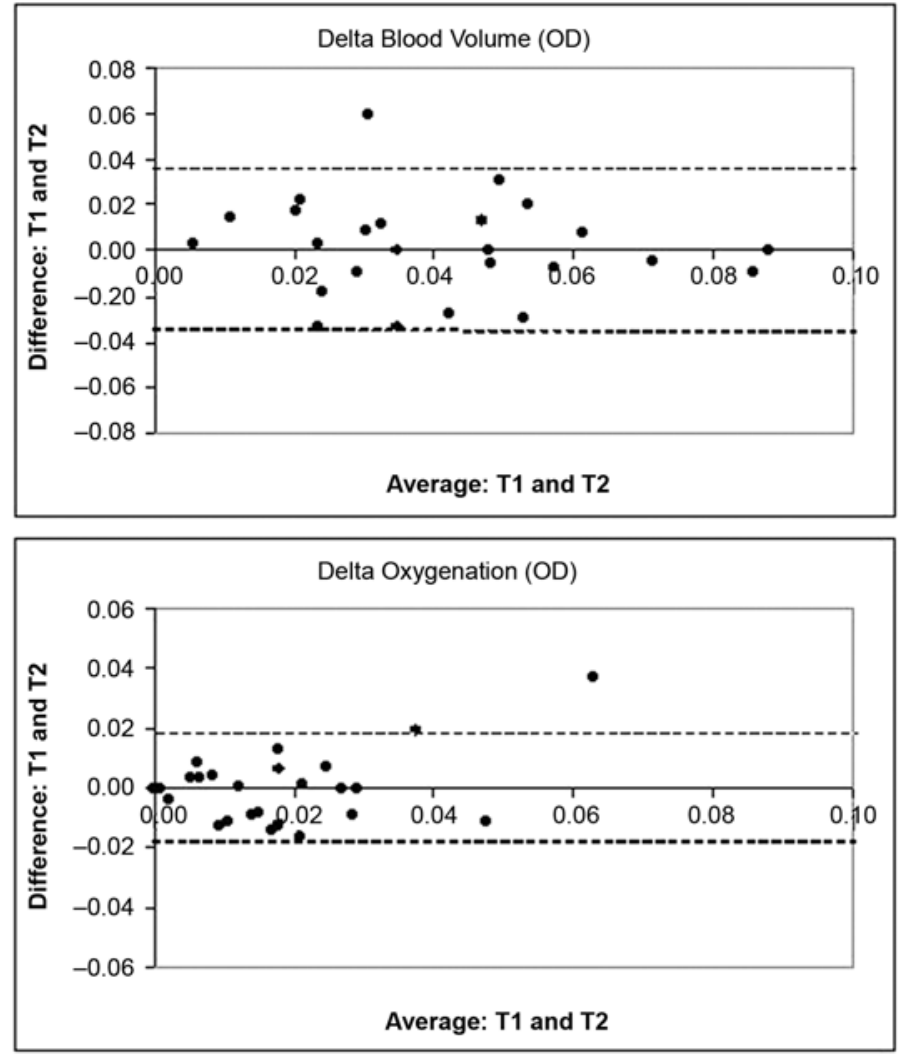

Figure 2.

Bland-Altman plots of changes in cerebral oxygenation and blood volume in (a) nondisabled subjects and (b) subjects with traumatic brain injury. Dashed lines indicate $95 \%$ confidence interval ( \pm 1.96 standard deviation). Data points above and below confidence interval were considered outliers. $\mathrm{OD}=$ optical density, $\mathrm{T} 1=$ trial $1, \mathrm{~T} 2=$ trial 2.

TBI were 58 and 36 percent, respectively. In general, the evidence indicates that (1) the reproducibility of the cerebral NIRS responses was moderately high in both subject groups and (2) these responses varied more in the subjects with TBI compared with their nondisabled counterparts.

\section{Comparison of Mean Cerebral Oxygenation and Blood Volume Responses Between Nondisabled and TBI subjects}

The mean cerebral oxygenation and blood volume responses during handgrip contractions in the nondisabled subjects and subjects with TBI are illustrated in Figure 4. The two-way analysis of variance with repeated measures indicated no significant group $\times$ trial interaction for either variable, implying that the nondisabled subjects and subjects with TBI did not significantly differ when the two trials were individually examined. However, examination of the main effects for cerebral oxygenation indicated sig- nificance for the group factor ( $p=0.038$ ) but not the trial factor $(p=0.164)$. The main effects for cerebral blood volume were not significant for either the group or the trial factors. These findings imply that when the values of the two trials were pooled, only cerebral oxygenation was significantly lower in the subjects with TBI compared with their nondisabled counterparts.

\section{DISCUSSION}

\section{Cerebral Oxygenation and Blood Volume Trends During Handgrip Contractions in Nondisabled and TBI Subjects}

Currently limited research has examined the use of NIRS for measuring alterations in cerebral oxygenation and blood volume during functional tasks. The observed increases in these two variables during the handgrip 
(a)
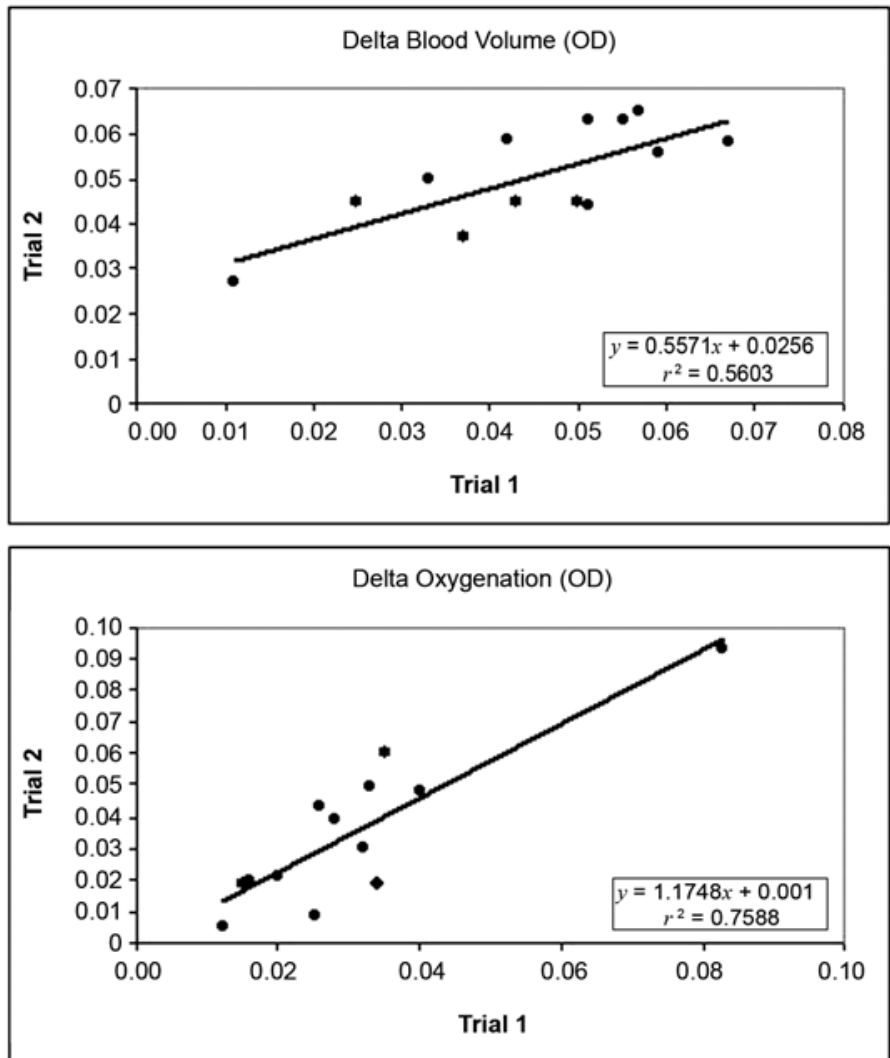

Figure 3.

Scatterplots comparing cerebral oxygenation and blood volume responses during two handgripping trials in (a) nondisabled subjects $(n=13)$ and (b) subjects with traumatic brain injury $(n=25)$. OD = optical density.

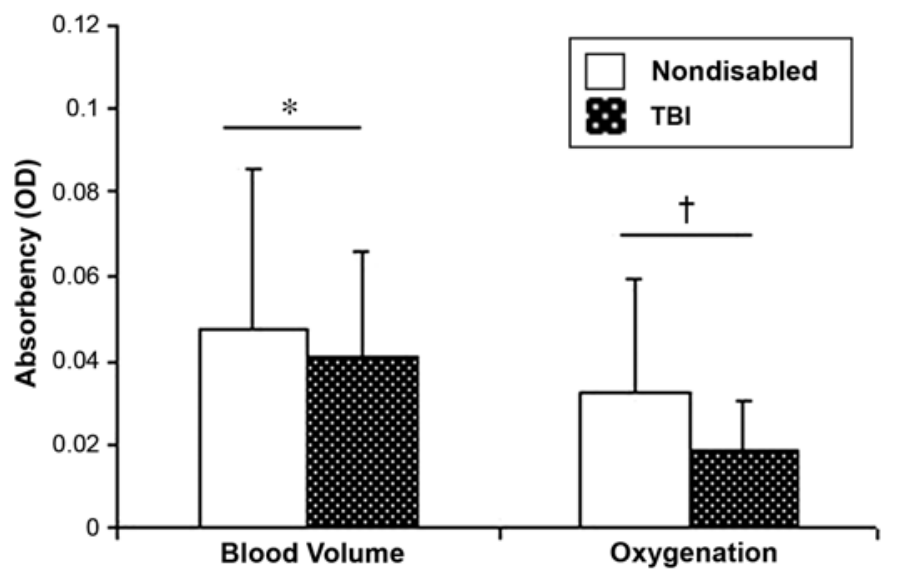

Figure 4.

Mean cerebral oxygenation and blood volume values during maximal intermittent handgrip contractions in nondisabled subjects and subjects with traumatic brain injury (TBI). Group mean values were average of two trials each group. ${ }^{*}$ Not significant, ${ }^{\dagger} p<0.05$. OD = optical density. (b)
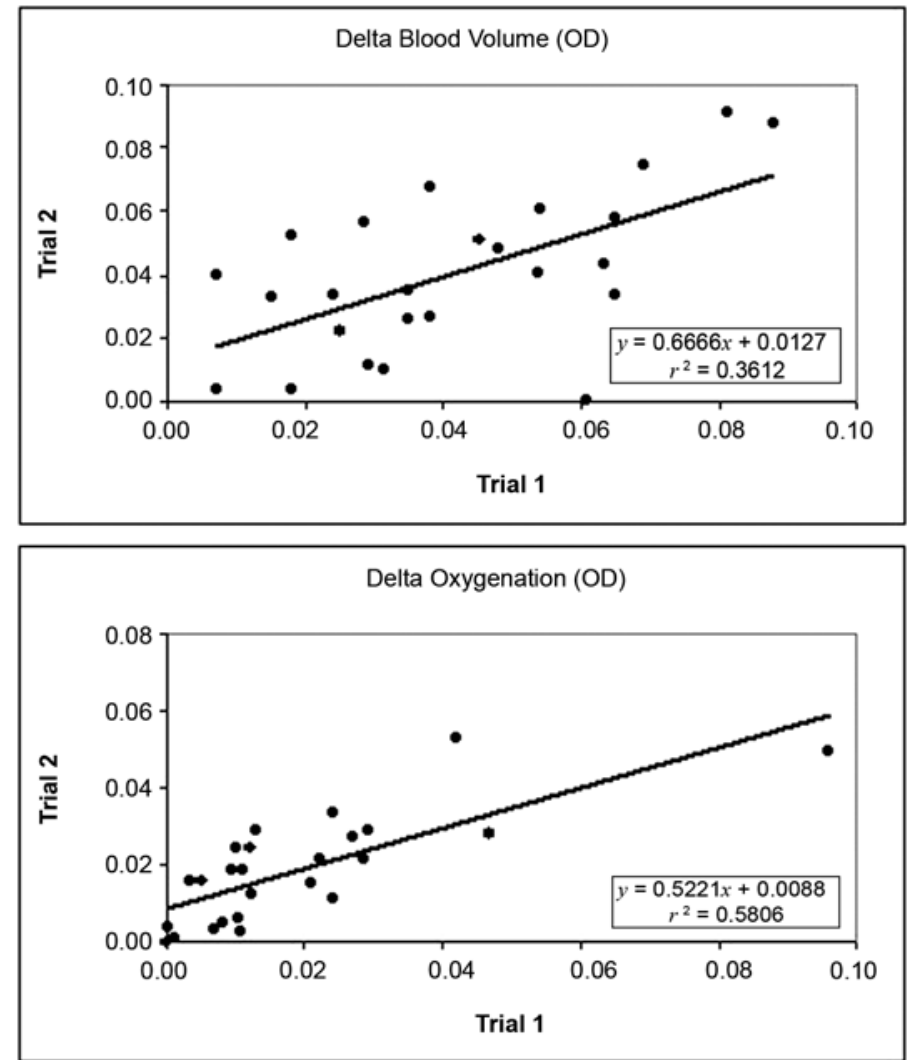
In the current study, we used intermittent handgrip contractions over a 1-minute interval to continually stimulate motor function and attain maximal cerebral activation. However, several subjects' cerebral oxygenation and cerebral blood volume values peaked before the end of the 1-minute interval and began to decline toward the resting value. These observations are consistent with the findings of Obrig et al. [13], who reported that several subjects' oxygenation and blood volume values peaked prior to termination of the 10-second finger opposition task. These findings collectively suggest an upper limit to the increases in cerebral oxygenation and blood volume for a given task. In all of our nondisabled subjects and subjects with TBI, the magnitude of the increase in cerebral blood volume was greater than that of cerebral oxygenation. This finding is also consistent with previous research $[13,15]$ and suggests that cerebral motor activation may depend more on localized blood flow than on neuronal oxygen extraction. Following the handgrip exercise, the cerebral oxygenation and cerebral blood volume returned toward the resting baseline values, and in most cases, the recovery was complete within the 2-minute period. However, in some instances, these responses were undershot (i.e., values decreased to below the baseline values), which was also documented by Obrig et al. [13]. Recent functional NIRS research has suggested that this undershoot, which is also evident in the BOLD signal during fMRI, may result from the persistent high oxygen consumption in the cerebral tissue after regional cerebral blood flow returns to baseline [32].

Currently, no studies have compared the cerebral NIRS responses during functional tasks in nondisabled subjects and subjects with chronic TBI. Our observations indicated that the general response pattern was similar in both groups during handgrip contractions. However, some subjects with TBI demonstrated increases in cerebral oxygenation and blood volume that were delayed by 5 to 10 seconds compared with the nondisabled subjects. This could possibly reflect delayed neuronal activation in the subjects with TBI. One should noted that Sakatani et al. reported significant differences between nondisabled adults and patients with cerebral ischemia and brain tumors for the cerebral oxygenation response measured by NIRS during a contralateral handgrip task [22].

\section{Reliability of Cerebral Oxygenation and Blood Volume Changes in Nondisabled and TBI Subjects}

Studies that have examined the test-retest reliability of cerebral NIRS responses are summarized in Table 2. A variety of experimental protocols and statistical methods have been used to demonstrate reliability, thereby making direct comparisons among the studies difficult. In some cases, reliability coefficients were not reported and the results were reported as reproducibility errors [6] or as a mean difference [7]. The general consensus from these findings is that the changes in cerebral oxygenation and blood volume were reproducible in younger and older males and females. To our knowledge, our study is the first to evaluate the reliability of the cerebral NIRS responses in nondisabled subjects and subjects with TBI during motor stimulation. Our findings demonstrate that the cerebral oxygenation and blood volume changes during maximal rhythmic handgrip contractions can be consistently reproduced in the subjects with TBI. The ICCs for cerebral oxygenation and blood volume were 0.83 and 0.80 , respectively, in the nondisabled subjects and 0.70 and 0.64 , respectively, in the subjects with TBI. The Bland-Altman analysis [29] revealed no outliers for these two variables in the nondisabled subjects (Figure 2(a)) but one outlier for cerebral blood volume and two outliers for cerebral oxygenation in the subjects with TBI (Figure 2(b)) during the handgrip contractions. The latter response could be due to differences in intracranial pressure in the subjects with TBI during the repeated trials [20]. These observations are fairly consistent with previous reports that evaluated the reproducibility of NIRS responses in nondisabled subjects. Examination of Totaro et al.'s data revealed two outliers for the oxyHb and total $\mathrm{Hb}$ responses and one outlier for the deoxyHb response during 3 minutes of hypercapnea [10]. Koike et al. reported no outliers for the cerebral oxyHb response during a symptom-limited incremental cycle exercise test in cardiac patients [11].

\section{Comparison of Cerebral Oxygenation and Blood Volume Changes Between Nondisabled and TBI Subjects}

In the current study, we hypothesized that the changes in cerebral oxygenation and blood volume during the handgrip contractions would be significantly lower in the subjects with TBI than in their nondisabled counterparts. Our results indicated that this was true for the cerebral oxygenation but not the blood volume 
Table 2.

Comparison of reliability of cerebral oxygenation and blood volume responses using near-infrared spectroscopy (NIRS).

\begin{tabular}{|c|c|c|c|c|c|}
\hline Study & Subjects & Task & $\begin{array}{l}\text { Probe } \\
\text { Position }\end{array}$ & Variables and Reliability Measure & Comments \\
\hline $\begin{array}{l}\text { Houtman } \\
\text { et al. [1] }\end{array}$ & $\begin{array}{l}\text { Nondisabled: } \\
10 \mathrm{M}\end{array}$ & SUP then HUT & L Frontal & $\begin{array}{l}\text { Change in- } \\
\text { - OxyHb: Pooled reproducibility error }= \\
1.8 \mu \mathrm{mol} / \mathrm{L} \\
\text { • DeoxyHb, Pooled reproducibility error }= \\
1.0 \mu \mathrm{mol} / \mathrm{L} \\
\text { - Total } \mathrm{Hb}, \text { Pooled reproducibility error }= \\
1.9 \mu \mathrm{mol} / \mathrm{L}\end{array}$ & $\begin{array}{l}\text { Pooled reproducibility error calculated } \\
\text { for between \& within visits for each } \\
\text { variable. Results indicated small error } \\
\text { for deoxyHb \& larger errors for oxyHb } \\
\text { \& total Hb. }\end{array}$ \\
\hline $\begin{array}{l}\text { Koike } \\
\text { et al. [2] }\end{array}$ & $\begin{array}{l}12 \text { patients with } \\
\text { heart disease }\end{array}$ & $\begin{array}{l}\text { Incremental } \\
\text { exercise }\end{array}$ & L Frontal & $\begin{array}{l}\text { Change in oxyHb: Pearson } r=0.88 \\
\text { Bland-Altman analysis }\end{array}$ & $\begin{array}{l}2 \text { tests } 11.3 \pm 4.6 \text { days apart. Bland- } \\
\text { Altman: no outliers. }\end{array}$ \\
\hline $\begin{array}{l}\text { Kurihara } \\
\text { et al. [3] }\end{array}$ & Nondisabled: $5 \mathrm{M}$ & $\begin{array}{l}5 \text { min @90 } \\
\text { HUT, } 3 \text { min @6 } \\
\text { HDT }\end{array}$ & $\begin{array}{l}\text { Frontal } \\
\text { Bilateral }\end{array}$ & $\begin{array}{l}\text { Tissue oxygenation index: Analysis of } \\
\text { variance }\end{array}$ & $\begin{array}{l}3 \text { sessions on separate days. Significant } \\
\text { difference between } 3 \mathrm{~d} \text { session } \& 1 \mathrm{st} / 2 \mathrm{~d} \\
\text { sessions on left but not right side. }\end{array}$ \\
\hline $\begin{array}{l}\text { Totaro } \\
\text { et al. [5] }\end{array}$ & $\begin{array}{l}\text { Nondisabled: } \\
12 \mathrm{M}, 15 \mathrm{~F}\end{array}$ & $\begin{array}{l}\text { Breathing } 5 \% \\
\text { carbon dioxide for } \\
3 \text { min }\end{array}$ & R Frontal & $\begin{array}{l}\text { Change in- } \\
\text { - OxyHb reactivity: Pearson } r=0.68 \\
\text { - DeoxyHb reactivity: Pearson } r=0.76 \\
\text { - Total Hb reactivity: Pearson } r=0.21\end{array}$ & $\begin{array}{l}\text { Carbon dioxide reactivity indices cal- } \\
\text { culated as changes in NIRS variable } \\
\text { divided by absolute change in end-tidal } \\
\text { carbon dioxide concentration. Bland- } \\
\text { Altman: two outliers for each index. }\end{array}$ \\
\hline $\begin{array}{l}\text { Watanabe } \\
\text { et al. [6] }\end{array}$ & 5 Nondisabled & $\begin{array}{l}\text { Design fluency, } \\
\text { verbal fluency, } \\
\text { hyperventilation }\end{array}$ & $\begin{array}{l}\text { Frontal } \\
\text { Bilateral }\end{array}$ & $\begin{array}{l}\text { For each task, change in oxyHb \& total Hb: } \\
\text { - ICC }=0.42,0.42 \text { (design fluency) } \\
\text { - ICC }=0.87,0.76 \text { (verbal fluency) } \\
\cdot \text { ICC }=0.65,0.56 \text { (hyperventilation) }\end{array}$ & $\begin{array}{l}\text { Reliability of NIRS measures influ- } \\
\text { enced by spontaneous oscillations } \\
\text { which could be due to auto regulation of } \\
\text { blood vessels. }\end{array}$ \\
\hline
\end{tabular}

1. Houtman S, Colier WN, Hopman MT, Oeseburg B. Reproducibility of the alterations in circulation and cerebral oxygenation from supine rest to head-up tilt. Clin Physiol. 1999;19(2):169-77. [PMID: 10200899]

2. Koike A, Itoh H, Oohara R, Hoshimoto M, Tajima A, Aizawa T, Fu LT. Cerebral oxygenation during exercise in cardiac patients. Chest. 2004;125(1):182-90. [PMID: 14718439$]$

3. Kurihara K, Kikukawa A, Kobayashi A. Cerebral oxygenation monitor during head-up and -down tilt using near-infrared spatially resolved spectroscopy. Clin Physiol Funct Imaging. 2003;23(4):177-81. [PMID: 12914555]

4. Mehagnoul-Schipper DJ, Colier WN, Jansen RW. Reproducibility of orthostatic changes in cerebral oxygenation in healthy subjects aged 70 years or older. Clin Physiol. 2001;21(1):77-84. [PMID: 11168300]

5. Totaro R, Barattelli G, Quaresima V, Carolei A, Ferrari M. Evaluation of potential factors effecting measurement of cerebrovascular reactivity by near-infrared spectroscopy. Clin Sci (Lond). 1998;95(4):497-504. [PMID: 9748426]

6. Watanabe A, Matsuo K, Kato N, Kato T. Cerebrovascular response to cognitive tasks and hyperventilation measured by multi-channel near-infrared spectroscopy. J Neuropsychiatry Clin Neurosci. 2003;15(4):442-49. [PMID: 14627771]

deoxyHb = deoxygenated hemoglogin, $\mathrm{F}=$ female, $\mathrm{ICC}=$ intraclass correlation coefficient, $\mathrm{Hb}=$ hemoglobin, HDT $=$ head-down tilt, HUT $=$ head-up tilt, $\mathrm{L}=$ left, $\mathrm{M}=$ male, oxyHb = oxygenated hemoglobin, $\mathrm{R}=$ rest, $\mathrm{SUP}=$ supine rest, $\mathrm{TBI}=$ traumatic brain injury.

changes (Figure 4). Comparisons of these two variables between nondisabled subjects and subjects with TBI during functional tasks are unavailable. Therefore, a discussion of these responses in patients with other neurological disorders is warranted. Sakatani et al. reported that the evoked responses in cerebral blood oxygenation measured by NIRS during contralateral handgrip tasks in patients with cerebral ischemia and brain tumors differed significantly from those of nondisabled adults [22]. The primary difference between the two groups was that the nondisabled subjects demonstrated a significant decrease in deoxyHb during the task, whereas the patients with cerebral ischemia and brain tumors showed a significant increase. Fujiwara et al. simultaneously examined the NIRS and fMRI responses in patients with cerebral ischemia and brain tumors during repeated handgrasping trials (40 seconds of rest and 40 seconds of handgrasping repeated six times) [23]. 
Their findings revealed significantly different NIRS responses (oxyHb, deoxyHb, and total $\mathrm{Hb}$ ) between the lesion and nonlesion sides. On the nonlesion side, the motor task evoked significant increases in oxyHb and total $\mathrm{Hb}$ with a concomitant decrease in deoxyHb in all subjects. However, on the lesion side, the NIRS responses could be differentiated by the deoxyHb response: one group ( $n=5)$ demonstrated a consistent increase in this variable, whereas another group $(n=7)$ showed a decrease. The fMRI data indicated that the deoxyHb-decrease group demonstrated significantly greater BOLD activation volumes on the lesion side compared with the nonlesion side and was more consistent with the response observed in nondisabled adults. Their study did not have a nondisabled comparison group, and the patients included males and females.

The current results indicate that although the cerebral oxygenation and blood volume responses tended to increase similarly during the handgrip task, the two subject groups did not significantly differ in the cerebral blood volume response. Research has demonstrated that in nondisabled subjects, cerebral blood flow and volume increase in proportion to the intensity of the task and the amount of metabolically active brain tissue $[13,15]$. Theoretically, this should have resulted in a larger increase in cerebral blood volume in the nondisabled subjects, because of their significantly greater handgrip strength. The reason for this inconsistency is unclear, but it could be related to the differences in sex composition between the two subjects groups. Previous studies have demonstrated that cerebral blood flow during neural activity is significantly higher in women than men [33-35]. More specifically, Uzuner et al. reported greater blood flow in women than men at rest and during handgrip contractions [35]. Since we had proportionately more males in the TBI group compared with the nondisabled group (84\% vs $62 \%$, respectively), the differences in cerebral blood volume between the two groups may have been masked.

\section{CONCLUSIONS}

During a maximal rhythmic handgrip exercise with the right hand, subjects' cerebral oxygenation and blood volume measured by NIRS over the left frontal lobe increased in parallel from the resting baseline values. The magnitude of the increase in cerebral blood volume exceeded that of cerebral oxygenation in both subject groups. The test-retest reliability of the changes in these variables was moderately high in both subject groups, but greater variability was observed in the subjects with TBI compared with their nondisabled counterparts. Comparisons of the mean responses between the two groups indicated that the increase in cerebral oxygenation during handgrip contractions was significantly lower in the subjects with TBI but the change in blood volume was similar. A major limitation of these findings is that continuous wave NIRS cannot quantify the absolute oxygen consumption of cerebral tissue because the path length factor is unknown [13-14]. Since cerebral mitochondrial function is impaired in patients with TBI [36], the NIRS technique must be refined to overcome this limitation and thereby allow objective monitoring of changes in cerebral hemodynamics during brain injury rehabilitation programs.

\section{ACKNOWLEDGMENTS}

Dr. Maikala is now with Liberty Mutual Research Institute for Safety, Hopkinton, Massachusetts.

This material was based on work supported by the Alberta Heritage Foundation for Medical Research, Health Research Fund, project 199800289.

The authors have declared that no competing interests exist.

\section{REFERENCES}

1. Simonson SG, Piantadosi CA. Near-infrared spectroscopy. Clinical applications. Crit Care Clin. 1996;12(4):1019-29. [PMID: 8902382]

2. Villringer A, Planck J, Hock C, Schleinkofer L, Dirnagl U. Near infrared spectroscopy (NIRS): A new tool to study hemodynamic changes during activation of brain function in human adults. Neurosci Lett. 1993;154(1-2):101-4. [PMID: 8361619]

3. Pollard V, Prough DS, DeMelo AE, Deyo DJ, Uchida T, Stoddart HF. Validation in volunteers of a near-infrared spectroscope for monitoring brain oxygenation in vivo. Anesth Analg. 1996;82(2):269-77. [PMID: 8561326]

4. Kleinschmidt A, Obrig H, Requardt M, Merboldt KD, Dirnagl U, Villringer A, Frahm J. Simultaneous recording of cerebral blood oxygenation changes during human brain activation by magnetic resonance imaging and near-infrared spectroscopy. J Cereb Blood Flow Metab. 1996;16(5): 817-26. [PMID: 8784226] 
5. Mehagnoul-Schipper DJ, Van der Kallen BF, Colier WN, Van der Sluijs MC, Van Erning LJ, Thijssen HO, Oeseburg B, Hoefnagels WH, Jansen RW. Simultaneous measurements of cerebral oxygenation changes during brain activation by near-infrared spectroscopy and functional magnetic resonance imaging in healthy young and elderly subjects. Hum Brain Mapp. 2002;16(1):14-23. [PMID: 11870923]

6. Houtman S, Colier WN, Hopman MT, Oeseburg B. Reproducibility of the alterations in circulation and cerebral oxygenation from supine rest to head-up tilt. Clin Physiol. 1999;19(2):169-77. [PMID: 10200899]

7. Kurihara K, Kikukawa A, Kobayashi A. Cerebral oxygenation monitor during head-up and -down tilt using nearinfrared spatially resolved spectroscopy. Clin Physiol Funct Imaging. 2003;23(4):177-81. [PMID: 12914555]

8. Mehagnoul-Schipper DJ, Colier WN, Jansen RW. Reproducibility of orthostatic changes in cerebral oxygenation in healthy subjects aged 70 years or older. Clin Physiol. 2001;21(1):77-84. [PMID: 11168300]

9. Watanabe A, Matsuo K, Kato N, Kato T. Cerebrovascular response to cognitive tasks and hyperventilation measured by multi-channel near-infrared spectroscopy. J Neuropsychiatry Clin Neurosci. 2003;15(4):442-49. [PMID: 14627771]

10. Totaro R, Barattelli G, Quaresima V, Carolei A, Ferrari M. Evaluation of potential factors affecting measurement of cerebrovascular reactivity by near-infrared spectroscopy. Clin Sci (Lond). 1998;95(4):497-504. [PMID: 9748426]

11. Koike A, Itoh H, Oohara R, Hoshimoto M, Tajima A, Aizawa T, Fu LT. Cerebral oxygenation during exercise in cardiac patients. Chest. 2004;125(1):182-90. [PMID: 14718439]

12. Fallgatter AJ, Strik WK. Frontal brain activation during the Wisconsin Card Sorting Test assessed with two-channel near-infrared spectroscopy. Eur Arch Psychiatry Clin Neurosci. 1998;248(5):245-49. [PMID: 9840371]

13. Obrig H, Hirth C, Junge-Hulsing JG, Doge C, Wolf $\mathrm{T}$, Dirnagl U, Villringer A. Cerebral oxygenation changes in response to motor stimulation. J Appl Physiol. 1996;81(3): 1174-83. [PMID: 8889751]

14. Obrig H, Villringer A. Near-infrared spectroscopy in functional activation studies. Can NIRS demonstrate cortical activation? Adv Exp Med Biol. 1997;413:113-27. [PMID: 9238492]

15. Colier WN, Quaresima V, Oeseburg B, Ferrari M. Human motor-cortex oxygenation changes induced by cyclic coupled movements of hand and foot. Exp Brain Res. 1999; 129(3):457-61. [PMID: 10591917]

16. Hirth C, Obrig H, Valdueza J, Dirnagl U, Villringer A. Simultaneous assessment of cerebral oxygenation and hemodynamics during a motor task. A combined near infrared and transcranial Doppler sonography study. Adv Exp Med Biol. 1997;411:461-69. [PMID: 9269463]
17. Adelson PD, Nemoto E, Colak A, Painter M. The use of near infrared spectroscopy (NIRS) in children after traumatic brain injury: A preliminary report. Acta Neurochir Suppl. 1988;71:250-54. [PMID: 9779198]

18. Buchner K, Meixensberger J, Dings J, Roosen K. Nearinfrared spectroscopy-not useful to monitor cerebral oxygenation after severe brain injury. Zentralbl Neurochir. 2000;61(2):69-73. [PMID: 10986754]

19. Cheng OS, Prowse S, Strong AJ. Oscillations in the nearinfrared signal in patients with severe head injury. Acta Neurochir Suppl. 2002;81:135-37. [PMID: 12168285]

20. Kampfl A, Pfausler B, Denchev D, Jaring HP, Schmutzhard E. Near infrared spectroscopy (NIRS) in patients with severe brain injury and elevated intracranial pressure. A pilot study. Acta Neurochir Suppl. 1997;70:112-14. [PMID: 9416295

21. Saitou H, Yanagi H, Hara S, Tsuchiya S, Tomura S. Cerebral blood volume and oxygenation among poststroke hemiplegic patients: Effects of 13 rehabilitation tasks measured by near-infrared spectroscopy. Arch Phys Med Rehabil. 2000;81(10):1348-56. [PMID: 11030500]

22. Sakatani K, Murata Y, Fukaya C, Yamamoto T, Katayama Y. BOLD functional MRI may overlook activation area in the damaged brain. Acta Neurochir Suppl. 2003;87:59-62. [PMID: 14518525]

23. Fujiwara N, Sakatani K, Katayama Y, Murata Y, Hoshino T, Fukaya C, Yamamoto T. Evoked-cerebral blood oxygenation changes in false-negative activations in BOLD contrast functional MRI of patients with brain tumors. Neuroimage 2004;21(4):1464-71. [PMID: 15050571]

24. Gray DS. Slow-to-recover severe traumatic brain injury: A review of outcomes and rehabilitation effectiveness. Brain Inj. 2000;14(11):1003-14. [PMID: 11104140]

25. Gray DS, Burnham RS. Preliminary outcome analysis of a long-term rehabilitation program for severe acquired brain injury. Arch Phys Med Rehabil. 2000;81(11):1447-56. [PMID: 11083347]

26. Jennett B, Snoek J, Bond MR, Brooks N. Disability after severe head injury: Observations on the use of the Glasgow Outcome Scale. J Neurol Neurosurg Psychiatry. 1981;44(4): 285-93. [PMID: 6453957]

27. Roth M, Decety J, Raybaudi M, Massarelli R, DelonMartin C, Segebarth C, Morand S, Gemignani A, Decorps $\mathrm{M}$, Jeannerod M. Possible involvement of primary motor cortex in mentally simulated movement: A functional magnetic resonance imaging study. Neuroreport. 1996;7(7): 1280-84. [PMID: 8817549$]$

28. Keppel G. Design and analysis: A researcher's handbook. Englewood Cliffs (NJ): Prentice Hall Press; 1973.

29. Bland JM, Altman DG. Measuring agreement in method comparison studies. Stat Methods Med Res. 1999;8(2): 135-60. [PMID: 10501650] 
30. Giller CA, Giller AM, Cooper CR, Hatab MR. Evaluation of the cerebral hemodynamic response to rhythmic handgrip. J Appl Physiol. 2000;88(6):2205-13. [PMID: 10846037]

31. Kawashima R, Yamada K, Kinomura S, Yamaguchi T, Matsui H, Yoshioka S, Fukuda H. Regional cerebral blood flow changes of cortical motor areas and prefrontal areas in humans related to ipsilateral and contralateral hand movement. Brain Res. 1993;623(1):33-40. [PMID: 8221091]

32. Schroeter ML, Kupka T, Mildner T, Uludaq K, Von Cramon DY. Investigating the post-stimulus undershoot of the BOLD signal-A simultaneous fMRI and fNIRS study. Neuroimage. 2006;30(2):349-58. [PMID: 16257236]

33. Kastrup A, Li TQ, Glover GH, Kruger G, Moseley ME. Gender differences in cerebral blood flow and oxygenation responses during focal physiologic neural activity. J Cereb Blood Flow Metab. 1999;19(10):1066-71. [PMID: 10532630]

34. Rodriguez G, Warkentin S, Risberg J, Rosadini G. Sex differences in regional cerebral blood flow. J Cereb Blood Flow Metab. 1988;8(6):783-89. [PMID: 3192645]

35. Uzuner N, Yalcinbas O, Gucuyener V, Ozdemir G. Hand gripping effect on cerebral blood flow in normal subjects. Eur J Ultrasound. 2000;11(2):1147-50. [PMID: 10781664]

36. Verweij BH, Muizelaar JP, Vinas FC, Peterson PL, Xiong Y, Lee CP. Impaired cerebral mitochondrial function after traumatic brain injury in humans. J Neurosurg. 2000;93(5): 815-20. [PMID: 11059663]

Submitted for publication September 8, 2005. Accepted in revised form April 24, 2006. 